\title{
A SHARED DATABASE OF UNDERGROUND UTILITY LINES FOR 3D MAPPING AND GIS APPLICATIONS
}

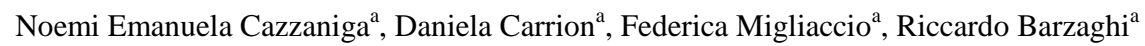

\author{
${ }^{a}$ Politecnico di Milano - DICA - Geodesy and Geomatics, Piazza Leonardo da Vinci 32, Milan, Italy \\ (noemi.cazzaniga,daniela.carrion,federica.migliaccio,riccardo.barzaghi)@ polimi.it
}

KEY WORDS: GIS, relational database, underground utility lines

\begin{abstract}
:
For the purpose of facility management it is very important to have detailed and up-to-date databases of underground utility lines, but such data are not always available with adequate accuracy. Hence, the need of collecting and organizing suitable information on underground services is a fundamental issue when dealing with urban data. Besides, by analyzing the process of designing and laying new underground infrastructures it is possible to implement an efficient and cost-effective approach to integrate and update existing maps by exploiting the surveying required for the installation of new facilities. It is also important to underline that collecting all the data in a unique integrated database (and GIS) gives the possibility to share (at least at a local level) the cartographic and thematic information for an optimal management of underground networks. In this paper, a database (DB) model for archiving the underground lines data is presented. The structure of the DB has been designed by following the standard methodology for the modelling of a relational DB, going through successive phases and originating the external, conceptual and logical model. Finally, preliminary tests have been carried on for parts of the DB to verify quality parameters.
\end{abstract}

\section{INTRODUCTION}

The availability of detailed and up-to-date cartographic representations of underground utility lines (as water supply, sewage system, electrical grid, gas network, district heating network, telecommunications and wiring network, oil pipelines) is very useful for network facility management, both for private companies and for local administrations. The knowledge of the 3D position and of the characteristics of the underground services is fundamental to be able to maintain the existing networks and to plan the installation of new ones, as it has been remarked in Blachut et al. (1979). Looking at the scientific literature, there are some examples of dealing with underground networks from the point of view of a DB design; in most cases the GIS environment is only exploited from the cartographic or computational point of view (Wang et al., 2012; Olivera et al., 2006). In $\mathrm{Du}$ et al. (2006) a suggestion for 3D pipelines visualization in a DBMS environment (Oracle Spatial) is presented, nevertheless in this case the focus is on the management of the geometry: in the proposed approach, the elements are represented as lines and this choice is compared with others (e.g. cylindrical or rectangular representation). A $\mathrm{DB}$ model is presented in $\mathrm{Lu}$ (2011) for the $\mathrm{Xi}^{\text {'an city gas }}$ company, where geometric, maintenance and interface with the public aspects are taken into account.

Unluckily, at the moment, the information on underground utility lines and of other buried objects is often incomplete and above all has an insufficient accuracy for land navigation of trenchers, to dig and lay cables. Regarding maps of the underground, even nowadays it is not very common to have exhaustive accurate information on position of underground utilities, and in this respect Italy shows a relevant gap. In many cases only maps with qualitative positioning of cables and pipelines are available. At the European level, in the framework of the INSPIRE directive, the technical specifications for utility services have been set in (INSPIRE, 2013). However in this approach "the level of details [...] is less important than collecting exhaustively the same type of data for the whole territory analysed" (ibid., p.22). At the Italian national level,
D.P.C.M. 2011 follows the same approach, defining the contents and models to be adopted in GIS by public administrations.

To obtain a sufficiently accurate and complete representation of the underground, a dedicated and extended survey is required. Non-invasive surveying can be performed with traditional topographic methods or by GPS positioning, by measuring the positions of the surface elements of the networks, like manholes, catch basins, transformers, hydrants, exchange boxes, etc., and subsequently inferring the presence and the approximate planar location of the buried lines. Another possibility is the GPR (Ground Penetrating Radar) technique (Metje et al., 2007), which allows reconstructing the main 3D geometry of underground elements. But, despite the major advantage of exploring the underground with trenchless means, there are limitations to the GPR technology linked to soil type and saturation and to the utility depth; besides, performing the survey of a large area is an expensive solution.

By analysing the process of designing and laying new infrastructures, it is evident that at different stages updates of such data are feasible. Hence, an efficient and cost-effective approach could be exploiting the surveying already required for the installation of new lines, in order to update the existing maps with data regarding both the new and the existing lines. In this paper a relational DB model is presented to store and manage the information related to designing and laying of underground utility services, taking into account the existing cartography and its update, the excavation plan and operations.

\section{ANALYSIS OF THE PROCESS OF DESIGNING AND LAYING AN UNDERGROUND UTILITY LINE}

The laying of underground utilities is a complex process which involves different actors, such as the network owner, the company who lays the network, GIS and surveying operators. The input to the process is the initially known information: the assignment, the above-ground map and the existing map of underground, if available. 
This input information is necessary to prepare a preliminary project of the laying that, again, allows to plan successive surveying activities, aimed at describing the underground preexisting elements, such as topographic or GPS and GPR surveying. The acquired information allows the design of the final project. Then, it is possible to obtain the mandatory legal permissions and to plan the digging and laying operations, including the definition of the excavation path.

During the digging operations, when traditional trenching techniques are adopted, also the physical exploration of the underground is possible, so in this case the underground utility map can be directly updated. When other types of trenchers are used, the laying of the facility lines can be performed at the same time of the digging operations, including the filling of the excavation, so in this case the direct observation of the existing lines is not possible.

The output of the process is constituted by

- cartographic representation of the new laid elements;

- up-to-date cartographic representation of the pre-existing underground services (and often of other buried objects).

While designing the GIS of underground utility networks, it has been considered a basic issue to adhere to standard procedures and formats when collecting data to obtain a consistent digital archive and an interoperable GIS. The aim is to model a shared DB containing all information regarding the underground utility lines, including both geo-referenced data and other attributes which are fundamental in the monitoring and maintaining of such networks.

An important result is that data of the underground utilities can be shared among different companies and collected in a unique integrated DB (and GIS) for the management at the level of at least a single Municipality.

As already experienced in previous projects (Carrion et al., 2009), the structure of the DB has been designed by following the standard methodology defined by ANSI (American National Standards Institute) SPARC Committee (Standards Planning And Requirements Committee), see also (Jardine, 1977).

A fundamental question concerning the modelling of the DB regards the level (or levels) at which data should be acquired and archived in the DB. The moments at which updates of the underground services mapping data occur can be detected by analysing the process of designing and laying pipes and cables. The data collected at such stages can effectively contribute to the information content of the DB. Following this strategy, the external model was established. The input and output data so defined are very heterogeneous and can be grouped into four categories:

- already existing cartographic data, which are partially organized as a DB and partially represented by graphic layers without attributes;

- data related to GPR surveys, i.e. raw data both collected during the survey operations and resulting from their processing (3D tomographic images, often geo-referenced in a local frame), in proprietary file format;

- positioning data, not always specifically characterized as mapping data and usually stored in various formats (proprietary or standard ASCII file format), strictly depending on the used type of instrument;

- data concerning the design phases of the new underground utility elements; different types of data can be involved, since every company may freely choose the formats which they consider best fitted for their aims (usually CAD files).

\section{THE CONCEPTUAL MODEL FOR A SHARED DB OF UNDERGROUND UTILITY NETWORKS}

In this phase of the DB modelling, a "bottom-up" strategy has been followed, identifying four main elements which can contribute to the structure of the DB itself: design, survey, navigation for digging purposes and final design of the network. As a consequence, the ERM (Entity Relationship Model) has been designed based on those four elements, which have been later integrated in a unique conceptual scheme of the DB.

Two different types of entities were included in the ERM: nongeometric and geometric entities, both with attributes. In the latter case, elements can have point or polyline geometry, following the guidelines issued by (D.P.C.M. 2011). However, also entities with polygon geometry have been included in the DB design, since the necessity could arise to describe objects which belong to networks and have not negligible dimensions, such as wet ponds or wells. The (D.P.C.M. 2011) regulations have been also taken into account when defining the attributes of the elements of underground lines.

The four main elements of the DB model are the following:

- Laying design: this part of the DB model is intended to store the data of the project and the information used to design it, as existing maps and urban plans.

- Survey of the existing network: this part of the ERM regards the data obtained from the GPR: raw data of GPS or topographic instrument used for geo-referencing the GPR and the results of their post-processing (usually, as a minimum, positions and mean square errors), and the GPR data (raw data and 3D images). Furthermore, it is useful to define two geometric entities: one with linear geometry and one with polygonal geometry. In both cases, an attribute represents the path to the original 3D GPR image file from which the data were extracted, for traceability purposes. The positioning accuracy can be associated to the entities in a specifically defined attribute. However, since the GPR is not able to determine the characteristics of the detected lines (material, diameter and so on), these two entities cannot be classified according to their intended use.

- Navigation for digging and laying purposes: data needed for the trencher navigation. In this case, one entity is defined to store general data regarding the navigation, one geometric entity (defined in the design phase) represents the route to be followed, and one geometric entity contains points useful for security purposes (e.g. start and stop points placed at a safe distance from other lines). Images (if photographic documentation is available) and navigation data are included in the DB in the same way as GPR images: an attribute contains the path to the directory where images and navigation data files are stored.

- Final design of the network: it regards the actual operations realized on the underground networks at the construction site. The company must supply the Municipality (INSPIRE, 2013) with the information on what elements have been altered or added to the existing network, and the final layout of the network could result to be different (although slightly) from the one planned in the design phase. This information can be obtained from the real time navigation data of the trencher and the design must comply with the regulations in force. 


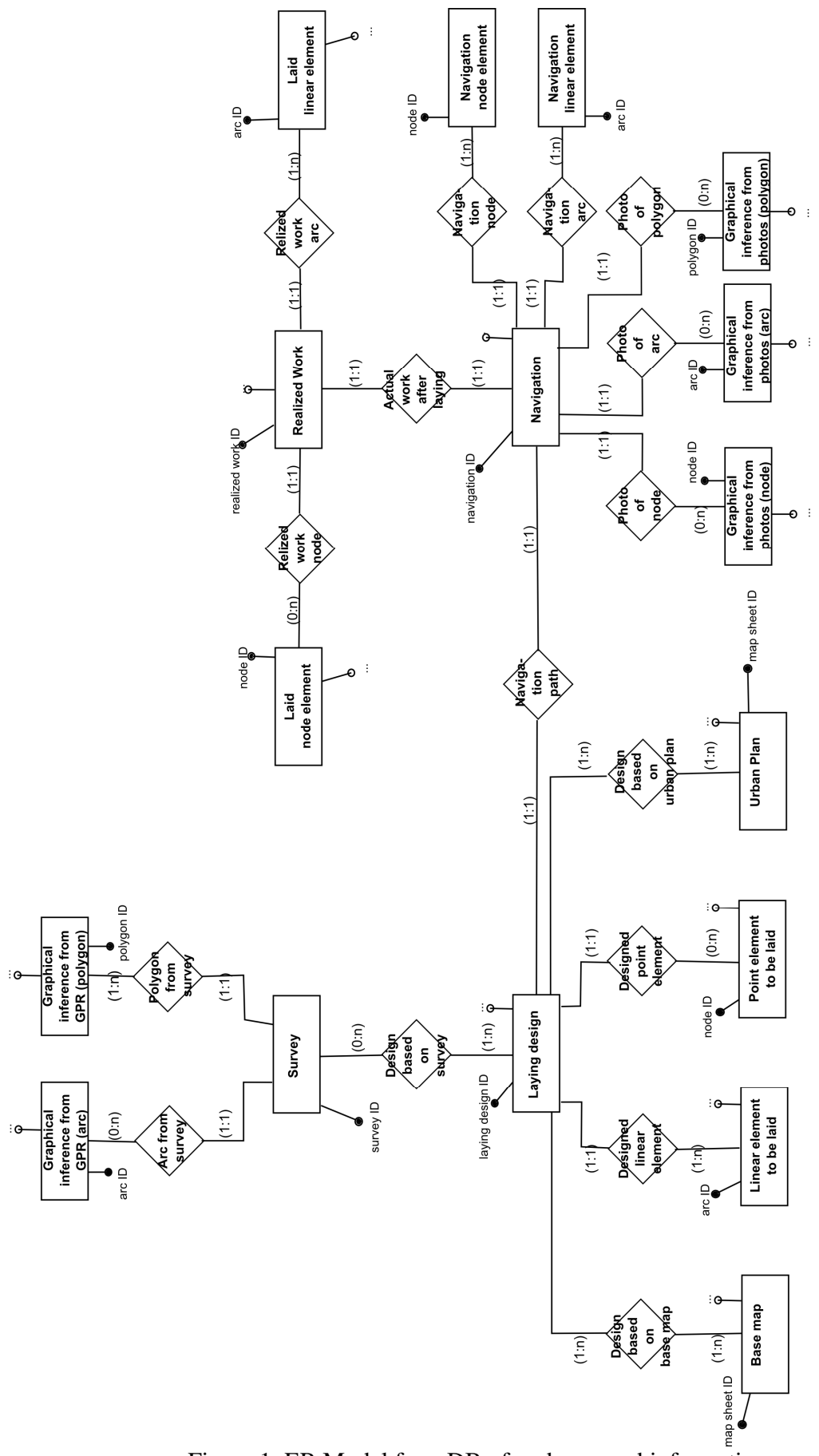

Figure 1. ER Model for a DB of underground information

The four elements described above have been integrated to obtain a unique ER model. For this purpose, it was necessary to define three suitable relationships to connect the different elements of the conceptual model (see Figure 1).

\section{PRELIMINARY TESTS OF THE DB MODEL}

To validate the designed DB model, it was decided to test its compliance with real digging operations for a pipeline laying under some streets of the central area of the City of Milan. The considered project, involving the laying of a new district heating line (two parallel pipes: one for the feed and one for the return), had been designed and realized by the Italian multiutility company A2A S.p.A. The GPR survey has been performed by the DICA Department of Politecnico di Milano.
Regarding the base maps, the urban area of Milan is represented in a large scale (1:1000) city map geo-referenced in the national reference system, and stored in CAD format. An additional larger scale (not specified) map representing the interior of the involved building, also stored in a CAD layer, was used. Concerning the existing information on the underground infrastructures, officially in the considered zone of Milan only the drainage and the water systems are (partially) described according to the conceptual model of the most recent regional laws. This information is freely available to any user as ESRI 2D shapefile. A more detailed description of the existing surface objects could be gathered from other sources (e.g. a CAD file), or partially inferred (at least in a plausible range) by considering the regulatory requirements. 
To design the laying of the new heating line, the urban plans (PGT and PUGSS) were taken into account. Also this information (represented by a $p d f$ file) was stored according to the proposed DB model, archiving the identifier of the file and its storage location. The design phase has been carried on using CAD software and afterwards the information has been structured as required by the ERM; the lines and points of the district heating pipes were now stored in two separate 3D shapefiles.

After this step, the GPR survey has been performed and the raw data have been processed to reconstruct a $3 \mathrm{D}$ image of the underground, geo-referenced in a national reference system and in vector format. The results have been converted into shapefile format (see Figure 2). The GPR survey allowed to detect both polygonal and linear entities not present in the existing maps.

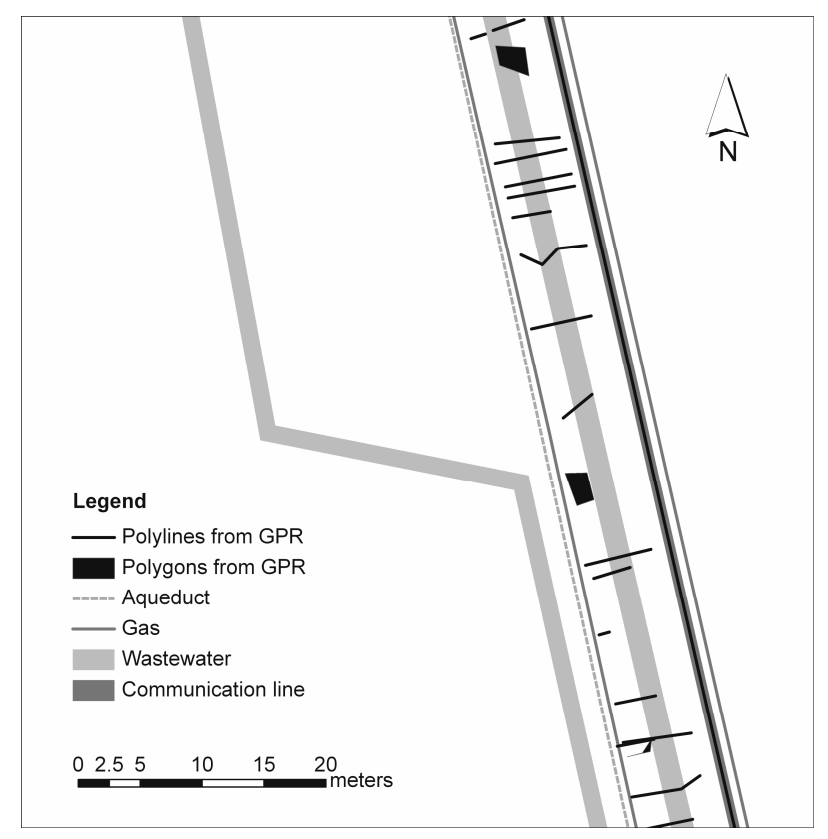

Figure 2. Portion of the 3D shapefiles of "inference from GPR" (black elements) superimposed to previously known underground information.

Besides, other entities appeared to be intersecting (in orthogonal projection) some already mapped lines; however they did not impact on the planned laying. The excavation for the realization of the project has been performed with traditional methods and the cables were laid according to the design. Since the preliminary project had already been converted in a format consistent with the DB requirements, the entities representing the realized work were consistent as well. Finally, this format is the one required by the regional law, hence the obtained files can be directly transmitted to the Municipality.

\section{CONCLUSIONS}

The work described in this paper regards the implementation of a geographical DB for the storage of data of underground structures and infrastructures. The structure of the DB has been designed based on an approach oriented to the study of the whole process of designing and laying new elements of a utility line. The results are compliant with the requirements of Italian national regulations. This approach allows a good traceability of all collected and archived data, giving the possibility to reconstruct the complete processing chain from the preliminary design of the utility lines to their laying. Regarding the geometric entities, it is important to remark that for all of them also the positioning precision has been archived: this is fundamental in order to consistently merge data coming from different sources (and consequently having different accuracies). The integration of such different data is still an open question, which will be investigated in the future.

\section{ACKNOWLEDGMENTS}

The Authors wish to thank A2A S.p.A for supplying data used for testing the DB structure; Maurizio Lualdi and Maurizio Porcu (DICA - Politecnico di Milano) for GPR data and data processing; Francesco Rosti, Erika lo Basso and Renata De Mitri for their contribution.

\section{REFERENCES}

References from Journals:

Carrion, D., Maffeis, A., Migliaccio, F. 2009. A DB oriented approach to GIS designing, Applied Geomatics, 1: 75-84.

Chen, P. 1976. The Entity-Relationship Model - Toward a Unified View of Data. ACM Transactions on DB Systems 1 (1): 9-36.

Codd, E.F. 1970. A Relational Model of Data for Large Shared Data Banks. Communications of the ACM 13 (6): 377-387.

Lu, L. 2011. DB Design Base on GIS Gas management Network, Procedia Engineering 15: 3954-3958.

Metje, N., Atkins, P.R., Brennan, M.J., Chapman, D.N., Lim, H.M., Machell, J., Muggleton, J.M., Pennock, S.R., Ratcliffe, J., Redfern, M.A., Rogers, C.D.F., Saul, A.J., Shan, Q., Swingler, S.G. \& Thomas, A.M. 2007. Mapping the Underworld. State of the Art Review. Tunnelling and Underground Space Technology 22(5-6): 568-586.

Olivera, F., Koka, S. \& Nelson, J. 2006. WaterNet: A GIS Application for the Analysis of Hydrologic Networks Using Vector Spatial Data, Transactions in GIS 10: 355-375.

Wang, Q., Wang Y., Zhang Q., Li D., Jie P. \& Shi Y. 2012. Establishment of the Model for Pipeline Network in DHS Based on MapGIS, 2nd International Conference on Remote Sensing, Environment and Transportation Engineering (RSETE), 2012: 1-4.

\section{References from Books:}

Blachut, T.J., Chrzanowski A. \& Saastamoinen J.H. 1979. Urban Surveying and Mapping: 221-234. Springer-Verlag, New York (USA).

Jardine, D.A. 1977. The ANSI-SPARC DBMS Model, NorthHolland Publishing Co. Amsterdam.

\section{References from Other Literature:}

D.P.C.M. 10 novembre 2011. "Regole tecniche per la definizione delle specifiche di contenuto dei DB geotopografici”, Gazzetta Ufficiale della Repubblica Italiana n.46, 27 febbraio 2011. (in italian)

Du, Y., Zlatanova, S. \& Liu, X. 2006. Management and 3D visualization of pipeline networks using DBMS and AEC software, Isprs commission IV symposium on geospatial DBs for sustainable development 34: 395-400.

\section{References from websites:}

INSPIRE. 2013. Data Specification on Utility and governmental services - Draft Technical Guidelines (2013-02-04). http://inspire.jrc.ec.europa.eu/documents/Data_Specifications/I NSPIRE_DataSpecification_US_v3.0rc3.pdf 